\title{
Perinatal asphyxia and its associated factors in Ethiopia: a systematic review and meta- analysis
}

Fikadu Waltengus Sendeku* , Getnet Gedefaw Azeze ${ }^{2}$ and Selamawit Lake Fenta ${ }^{1}$

\begin{abstract}
Background: Despite different preventive strategies that have been implemented in different health institutions in the country, neonatal mortality and morbidity are still significantly increasing in Ethiopia. Perinatal asphyxia is the leading cause of neonatal morbidity and mortality worldwide. As a result, this systematic review and meta-analysis aimed to assess the prevalence and associated factors of perinatal asphyxia in Ethiopia.

Methods: Online databases (PubMed, HINARI, EMBASE, Google Scholar and African Journals), other gray and online repository accessed studies were searched using different search engines. Newcastle-Ottawa Quality Assessment Scale (NOS) was used for critical appraisal of studies. The analysis was done using STATA 11 software. The Cochran $\mathrm{Q}$ test and $\mathrm{I}^{2}$ test statistics were used to test the heterogeneity of studies. The funnel plot and Egger's test were used to detect publication bias of the studies. The pooled prevalence of perinatal asphyxia and the odds ratio (OR) with a 95\% confidence interval was presented using forest plots.

Result: Nine studies were included in this review, with a total of 12,249 live births in Ethiopia. The overall pooled prevalence of perinatal asphyxia in Ethiopia was 24.06\% (95 95\%Cl: 18.11-30.01). Associated factors of perinatal asphyxia included prolonged labor $(\mathrm{OR}=2.79,95 \% \mathrm{Cl}: 1.98,3.93)$, low birth weight $(\mathrm{OR}=6.52,95 \% \mathrm{Cl}: 4.40,9.65)$, meconium-stained amniotic fluid $(\mathrm{OR}=5.91,95 \% \mathrm{Cl}: 3.95,8.83)$ and instrumental delivery $(\mathrm{OR}=4.04,95 \% \mathrm{Cl}: 2.48$, 6.60) were the determinant factors of perinatal asphyxia in Ethiopia.

Conclusions: The overall pooled prevalence of perinatal asphyxia was remarkably high. Duration of labor, meconium-stained amniotic fluid, instrumental deliveries, and birth weight were the associated factors of perinatal asphyxia in Ethiopia. Therefore, efforts should be made to improve the quality of intrapartum care service to prevent prolonged labor and fetal complications and to identify and make a strict follow up of mothers with meconium-stained amniotic fluid. This finding is important to early recognition and management of its contributing factors, might modify hypoxic-ischemic encephalopathy and may improve the implementation of the standard guideline effectively and consistently.
\end{abstract}

Keywords: Birth outcomes, Neonatal, Meta-analysis, Morbidity, And mortality, Systemic review

\footnotetext{
*Correspondence: fek0015@gmail.com

${ }^{1}$ Department of Midwifery, College of Medicine and Health Sciences, Bahir Dar University, Bahir Dar, Ethiopia

Full list of author information is available at the end of the article
}

(c) The Author(s). 2020 Open Access This article is licensed under a Creative Commons Attribution 4.0 International License, which permits use, sharing, adaptation, distribution and reproduction in any medium or format, as long as you give appropriate credit to the original author(s) and the source, provide a link to the Creative Commons licence, and indicate if changes were made. The images or other third party material in this article are included in the article's Creative Commons licence, unless indicated otherwise in a credit line to the material. If material is not included in the article's Creative Commons licence and your intended use is not permitted by statutory regulation or exceeds the permitted use, you will need to obtain permission directly from the copyright holder. To view a copy of this licence, visit http://creativecommons.org/licenses/by/4.0/ The Creative Commons Public Domain Dedication waiver (http://creativecommons.org/publicdomain/zero/1.0/) applies to the data made available in this article, unless otherwise stated in a credit line to the data. 


\section{Background}

Perinatal asphyxia is the failure of neonates to initiate and sustain breathing at birth which causes significant neonatal morbidity and mortality to the extent of the first week of life [1]. Perinatal asphyxia is a condition that occurs when there is an impairment of blood gas exchange that results in hypoxemia and hypercapnia. Moreover, perinatal asphyxia requires adequate and quick resuscitation measures $[2,3]$. The combination of hypoxia and ischemia will result in a cascade of biochemical changes in the body leading to brain neuronal death and brain damage during the antepartum or intrapartum period [4].

According to the World Health Organization (WHO), there were nearly four to nine million newborns develop birth asphyxia each year. APGAR score is used to determine the level of perinatal asphyxia, evaluated in the 1st and 5thminutes of life with scores ranging from zero to ten. Four to seven APGAR score in the first minute of life indicates moderate perinatal asphyxia and between zero and three indicates severe asphyxia [5].

According to WHO, perinatal asphyxia is characterized by profound metabolic acidosis, $\mathrm{PH}<7.20$ on umbilical cord arterial blood sample, the persistence of an APGAR score of 3 at the 5 th minute, clinical neurologic sequelae in the immediate neonatal period, evidence of multi-organ system dysfunction (brain damage, heart, lungs, gut, kidneys) in the immediate neonatal period. Pre-pregnancy risk factors for perinatal asphyxia are; maternal age $\geq 35$ years, social factors, family history of neurologic disease, infertility treatment, previous neonatal death, chronic illness. Whereas antepartum risk factors include; maternal thyroid disease, severe preeclampsia, multiple gestation, chromosomal/genetic abnormalities, congenital malformations, intrauterine growth restriction, trauma, malpresentation, and antepartum hemorrhage. Besides, chorioamnionitis, meconium-stained amniotic fluid, operative vaginal delivery, general anesthesia, emergency cesarean delivery, placental abruption, cord accident, uterine rupture, maternal cardiac arrest, and fetal exsanguinations were the intrapartum risk factors for perinatal asphyxia $[3,6]$.

In low and middle-income countries neonatal mortality rate constitutes $42 \%$ of under- 5 deaths [1, 6 , 7]. According to the World Health Organization report, perinatal asphyxia is the third leading cause of under-5 child deaths (11\%) following preterm birth (17\%) and pneumonia (15\%) [2, 6, 8]. In low and middle-income countries, neonatal deaths accounted for $52 \%$ of all under -5 child mortality in
South Asia, 53\% in Latin America and Caribbean, and $34 \%$ in Sub-Saharan Africa due to preventable causes including perinatal asphyxia [9].

Aslam HMSS et. al [3] in Ethiopia, perinatal asphyxia is one of the leading causes of neonatal mortality, which accounts for 34\% [10]. Therefore, this systematic review and meta-analysis aimed to estimate the pooled prevalence of perinatal asphyxia and to identify the determinant factors in Ethiopia.

\section{Methods}

\section{Search strategy}

International Online databases (Pub Med, HINARI, EMBASE, Google Scholar and African Journals) were used to search articles on perinatal asphyxia. Searching terms were based on adapted PICO questions to search through the aforementioned databases to accesses all the important articles. Different Boolean operators have been established to have comprehensive datasets on perinatal asphyxia. MeSH engines including "perinatal asphyxia" OR "birth asphyxia OR "neonatal asphyxia," OR "meconium-stained amniotic fluid" OR" mode of delivery", OR "preterm birth delivery", OR "low birth weight" OR "Small for gestational age", OR "prolonged labor" OR "hypoxic-ischemic encephalopathy" AND related in Ethiopia were searched in the international online electronic database and online university repositories.

\section{Inclusion and exclusion criteria}

Both cross-sectional and case-control studies were incorporated. Studies reported the prevalence and/or associated factors, or determinant factors of perinatal or birth asphyxia or hypoxic-ischemic encephalopathy were included in this study. Only English language literature and research articles were included. Whereas articles without full text and abstract, duplicated studies, anonymous reports, and editorial reports were excluded.

\section{Data extraction and quality assessment}

After collecting findings from all databases, the articles were exported to Microsoft Excel spreadsheet. Two authors (GG \& FW) independently extracted the data and reviewed all the screened and included articles. Any disagreement was handled by the third reviewer (SL). After all, a consensus was reached through discussion between authors. NewcastleOttawa Quality Assessment Scale (NOS) for crosssectional and case-control studies is used to assess the methodological quality of a study and to determine the extent to which a study addressed the possibility of bias in its design, conduct, and analysis. 
All authors independently assessed the articles for inclusion in the review. All of the included articles scored (NOS) seven and more can be considered a "good" study and low risk. However, in this study, all articles were included since they scored more than seven and above of the NOS criteria [11].

\section{Outcome of measurement}

The measurement outcome of this study had two main outcome variables. Perinatal asphyxia or hypoxicischemic encephalopathy was the first outcome of the study whereas associated factors of perinatal asphyxia or hypoxic-ischemic encephalopathy was the second outcome of the study. The odds ratio was calculated for the common factors of the reported studies. The outcome of this study was to focus on single studies estimating the prevalence of perinatal asphyxia among live birth neonates.

\section{Hypoxic-ischemic encephalopathy/HIE/}

Hypoxic-ischemic encephalopathy in a newborn was defined as a 5-min Apgar score of less than 5 and evidence of metabolic acidosis associated with one or more of the following signs of neurologic dysfunction: depression of the level of consciousness, respiratory depression, abnormality of muscle tone, disturbances of cranial nerve function and seizures (in the first week of life) [12].

\section{Publication bias and heterogeneity}

The Cochrane $\mathrm{Q}$ test and $\mathrm{I}^{2}$ with its corresponding $p$ value were used to assess the heterogeneity of the study. A value of 25,50 , and $75 \%$ was used to declare the heterogeneity test as low, medium and high heterogeneity respectively. Funnel plot and Egger regression asymmetry tests were employed to assess the existence of publication bias. As a result, the random effect model of analysis was computed with the evidence of heterogeneity [11].

\section{Data analysis}

The data were entered using Microsoft Excel. This systematic review and meta-analysis were conducted using Stata 11 software. The estimated prevalence of each study was presented using forest plots with a 95\% confidence interval (CI). Moreover, subgroup analysis was computed using the study region and the sample size of the studies, with the evidence of heterogeneity.

\section{Results}

Characteristics of the included studies

234 studies were retrieved at Pub Med, HINARI, EMBASE, Google Scholar and African Journals and other gray and online repository accessed articles regarding the prevalence and risk factors of perinatal asphyxia in Ethiopia. After duplicates were expunged, 113 studies remained.

Out of the remaining 113 articles, 87 articles were excluded after review of their titles and abstracts. Therefore, 26 full-text articles were accessed and assessed for inclusion criteria, which resulted in the further exclusion of 17 articles primarily due to reason. As a result, 9 studies were met the inclusion criteria to undergo the final systematic review and meta-analysis (Fig. 1). This systematic review and meta-analysis consist of five crosssectional and four case-control studies. Studies were conducted from different regions of Ethiopia (Amhara, Oromia, SNNPR (South Nation Nationalities people and representatives), Tigray and Dire Dawa. Overall, this review included a total of 12,249 neonates in Ethiopia (Table 1).

\section{Epidemiology of perinatal asphyxia in Ethiopia}

A wide-ranging prevalence of perinatal asphyxia was observed across different studies included in this review. The overall pooled prevalence of perinatal asphyxia in Ethiopia was 24.06\% (95 95\%CI: 18.11$\left.30.01, \mathrm{I}^{2}=93.5 \%, p=<0.001\right)$ using a random effect model (Fig. 2).

\section{Subgroup analysis}

Subgroup analysis was conducted based on the study region and sample size. Accordingly, the overall prevalence of perinatal asphyxia by region showed a pooled prevalence of perinatal asphyxia in Amhara \& Tigray (Northern part of Ethiopia) was $25.39 \%$ (95\% CI $=17.84,32.94$, $\mathrm{I}^{2}=70 \%, p=0.046$ ) followed by SNNPR (South Nation Nationalities people representatives) + DD (Dire Dawa) + Oromia (Eastern part of Ethiopia) was $23.22 \%(95 \% \mathrm{CI}=$ 13.79, 32.65, $\mathrm{I}^{2}=96.6 \%, p-<0.001$ ) (Fig. 3). Regarding sample size, having less than 380 study participants was 24.87(10.35-39.4) with 95\%CI, $\mathrm{I}^{2}=95.6, P<=0.001$ ) (Fig. 4).

\section{Publication bias}

A funnel plot showed that symmetrical distribution and Egger test $=0.763$. Hence, there is no publication bias in the studies (Fig. 5).

\section{Determinants of perinatal asphyxia \\ Relationship between low birth weight and perinatal asphyxia}

Six studies were included in this category of metaanalysis [13-18]. Neonates born with low birth weight were 6.52 times (OR: 6.52, 95\% CI: 4.40, 9.65) more likely to develop perinatal asphyxia as compared to those born with normal weight. In 


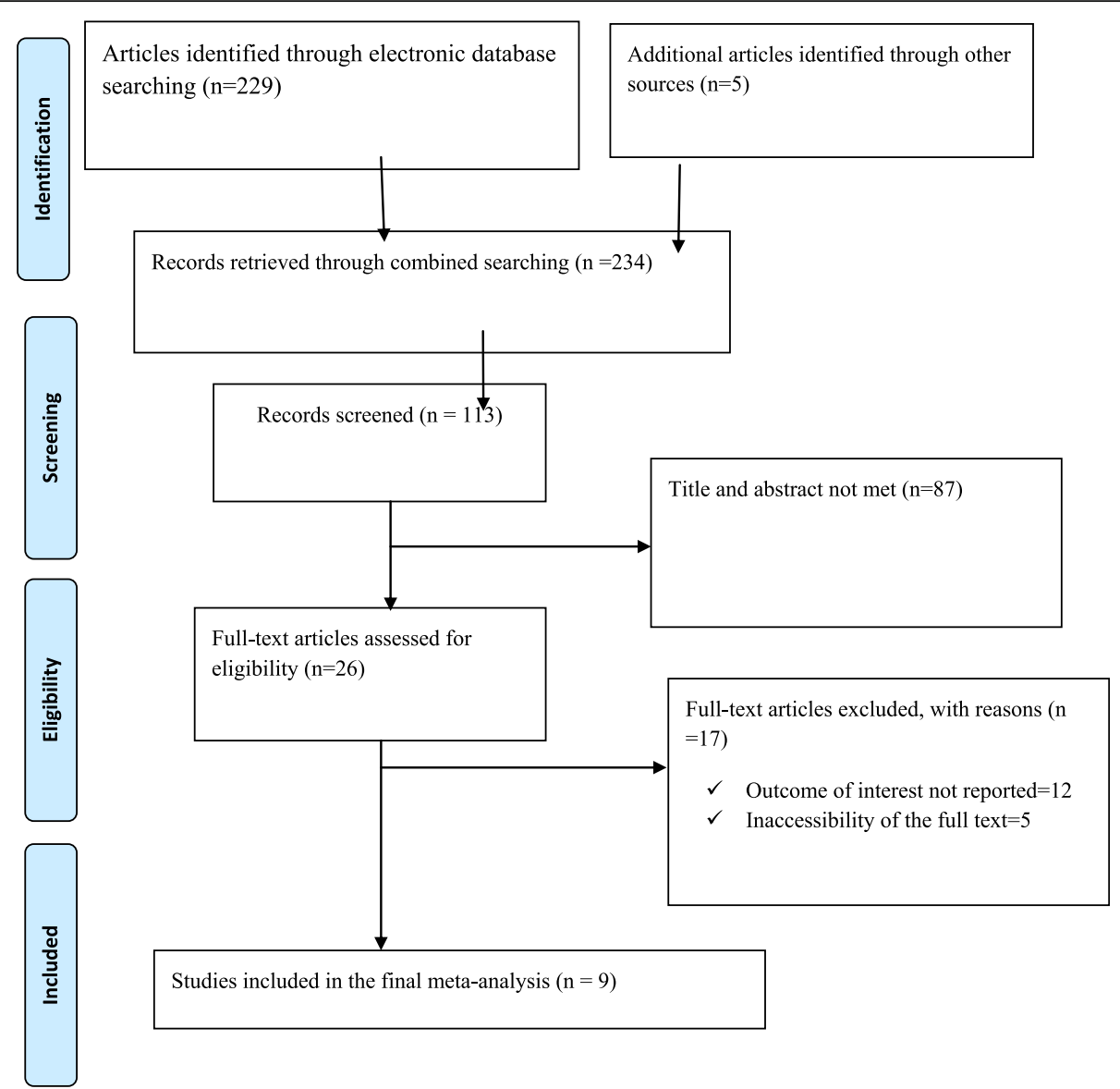

Fig. 1 PRISMA Flow chart of study selection for systematic review and meta-analysis of determinant factors of perinatal asphyxia in Ethiopia

this meta-analysis, included studies were characterized by no existence of heterogeneity $\left(\left(\mathrm{I}^{2}=\right.\right.$ $0.0 \%, P=0.441)$. Moreover, we used a fixed-effect model analysis due to the absence of heterogeneity (Fig. 6).

\section{Association between meconium-stained amniotic fluid and perinatal asphyxia}

Five studies were included in this category of metaanalysis [13-16, 18]. The likelihood of developing perinatal asphyxia among neonates born with meconium-stained amniotic fluid was 5.91 times $(\mathrm{OR}=$ 5.91, 95\% CI: 3.95, 8.83) more likely to develop birth asphyxia than the counterparts. In this meta-analysis, included studies were characterized by low heterogeneity $\left(\mathrm{I}^{2}=18.1 \%, P=0.300\right)$, meanwhile, we calculated a random effect meta-analysis (Fig. 7).

\section{Association between mode of delivery and perinatal asphyxia}

Three studies were included in this category of meta-analysis $[16,17,19]$. The likelihood of developing perinatal asphyxia among neonates born with assisted instrumental delivery was 4.04 times $(\mathrm{OR}=4.04,95 \% \mathrm{CI}: 2.48,6.60)$ more likely to develop birth asphyxia than neonates born with spontaneous delivery. In this meta-analysis, included studies were characterized by low heterogeneity $\left(\mathrm{I}^{2}=4.6 \%, P=0.350\right)$. Furthermore, we computed a random effect meta-analysis with the evidence of heterogeneity (Fig. 8).

\section{Association between duration of labor and perinatal asphyxia}

Five studies were included in this category of metaanalysis [13, 14, 16, 17, 19]. The odd of developing perinatal asphyxia among neonates born from mothers with prolonged labor was 2.79 times $(\mathrm{OR}=$ 2.79, 95\% CI: 1.98, 3.93) more compared with their counterparts. In this meta-analysis, included studies were characterized by no possibility of heterogeneity $\left(\mathrm{I}^{2}=0.0 \%, P=0.419\right)$. Therefore, we computed a fixed-effect meta-analysis with the evidence of heterogeneity of the studies (Fig. 9). 
Table 1 Study characteristics included in the systematic review and meta-analysis

\begin{tabular}{|c|c|c|c|c|c|c|c|c|}
\hline No & Authors & Study year & Study design & live births & Region & Gestational age & Study area & $\mathrm{P}(95 \% \mathrm{Cl})$ \\
\hline 1 & Yohannes K et al & 2018 & Case-control & 380 & Amhara & $\begin{array}{l}\text { Pre-term }=48 \\
\text { Term }=318 \\
\text { Post-term }=14\end{array}$ & $\begin{array}{l}\text { General \& referral } \\
\text { hospitals, } \\
\text { Dessie, Amhara } \\
\text { region }\end{array}$ & - \\
\hline 2 & Abebe A et al & 2017 & Cross-sectional & 256 & SNNPR & $\begin{array}{l}\text { Pre-term =66 } \\
\text { Term }=153 \\
\text { Post-term =33 }\end{array}$ & $\begin{array}{l}\text { Dilla Universty } \\
\text { referral hospital, } \\
\text { SNNPR }\end{array}$ & $\begin{array}{l}32.80 \\
(27.12,38.48)\end{array}$ \\
\hline 3 & Neil B et al & 2014-2017 & Cross-sectional & 9738 & Dire Dawa & $\begin{array}{l}\text { Pre-term }=36 \\
\text { Term }=210 \text { of } \\
\text { the total } 246 \\
\text { asphyxiated } \\
\text { babies }\end{array}$ & $\begin{array}{l}\text { Dilchora Referral } \\
\text { hospital, Dire Dawa }\end{array}$ & $\begin{array}{l}25.00 \\
(24.14,25.86)\end{array}$ \\
\hline 4 & Worku N et al & 2017 & Cross-sectional & 154 & Amhara & $\begin{array}{l}\text { Pre-term = } 20 \\
\text { Term \&post- } \\
\text { term }=134\end{array}$ & $\begin{array}{l}\text { General hospital } \\
\text { Amhara region }\end{array}$ & $\begin{array}{l}29.90 \\
(22.67,37.13)\end{array}$ \\
\hline 5 & Lisanu W et al & 2017 & Case-control & 270 & Amhara & $\begin{array}{l}\text { Pre-term }=27 \\
\text { Term }=217 \\
\text { Post-term }=26\end{array}$ & $\begin{array}{l}\text { Gondar referral } \\
\text { hospital, Amhara } \\
\text { region }\end{array}$ & - \\
\hline 6 & Zelalem J et al & 2015 & Cross-sectional & 371 & Oromia & $\begin{array}{l}\text { Reported only } \\
\text { live birth without } \\
\text { GA }\end{array}$ & $\begin{array}{l}\text { General, referral \& } \\
\text { primary hospitals, } \\
\text { Oromia region }\end{array}$ & $\begin{array}{l}12.50 \\
(9.13,15.87)\end{array}$ \\
\hline 7 & Gdiom G et al & 2018 & Cross -sectional & 421 & Tigray & $\begin{array}{l}\text { Pre-term }=25 \\
\text { Term }=396\end{array}$ & $\begin{array}{l}\text { General hospital } \\
\text { Tigray region }\end{array}$ & $\begin{array}{l}22.10 \\
(18.14,26.06)\end{array}$ \\
\hline 8 & Alemwork D et al & 2018 & Case-control & 386 & Amhara & $\begin{array}{l}\text { Pre-term = } 37 \\
\text { Term }=326 \\
\text { Post-term }=23\end{array}$ & $\begin{array}{l}\text { Amhara region } \\
\text { hospitals } \\
\text { General, referral } \\
\text { \& primary }\end{array}$ & - \\
\hline 9 & Hagos T et al & 2018 & Case-control & 264 & Tigray & $\begin{array}{l}\text { Pre-term }=89 \\
\text { Term }=175\end{array}$ & $\begin{array}{l}\text { General, referral \& } \\
\text { primary hospitals, } \\
\text { Tigray region }\end{array}$ & - \\
\hline
\end{tabular}

\section{Discussion}

According to the results of this meta-analysis, the pooled prevalence of perinatal asphyxia in Ethiopia was estimated to be $24.06 \%$ (95\% CI: 18.11, 30.01). Practicing standard guidelines during labor and delivery across different hospitals and health institutions could reduce this significant neonatal burden at the national level.

This study finding is in line with the study done in Nigeria reporting that, $30.1 \%$ [20] and Tanzania (21.1\%) [21] of the neonates were having perinatal asphyxia. The finding of this study is lower than the study conducted in Bangladesh which was estimated $56.9 \%$ [22]. The discrepancy might be due to the difference of good utilization of different intrapartum fetal monitoring equipment and tools, having qualified professional and quality of care given services to neonates in Ethiopia.

The finding of this systematic review is higher than the study conducted in Thailand, Malawi and Guinea accounted for $11.7 \%$ [23], 6.1\% [24] and $2.4 \%$ [25] respectively. This discrepancy might be due to socio-cultural, antenatal care service accessibility, and variability in maternal risk factor distribution. Besides, most of the studies included in this meta-analysis were conducted in hospitals and health centers which might increase the prevalence.

Neonates born with low birth weight were 6.52 times (OR: 6.52, 95\% CI: 4.40, 9.65) more likely to develop birth asphyxia as compared to neonates born with normal birth weight. Similar study findings were observed in different countries: Uganda [26], Indonesia [27], Pakistan [3], East Java [28] and Thailand [29]. This could be explained by because a high proportion of small babies might be pre-term that they might not have enough surfactant which might not prevent suffering from difficulty of breathing and developing difficulty in cardiopulmonary transition and subsequent birth asphyxia.

The likelihood of developing birth asphyxia among neonates born with meconium-stained amniotic fluid was 5.91 times $(\mathrm{OR}=5.91,95 \% \mathrm{CI}: 3.95,8.83)$ as likely to have birth asphyxia as those born with clear amniotic. This finding was consistent with the study 


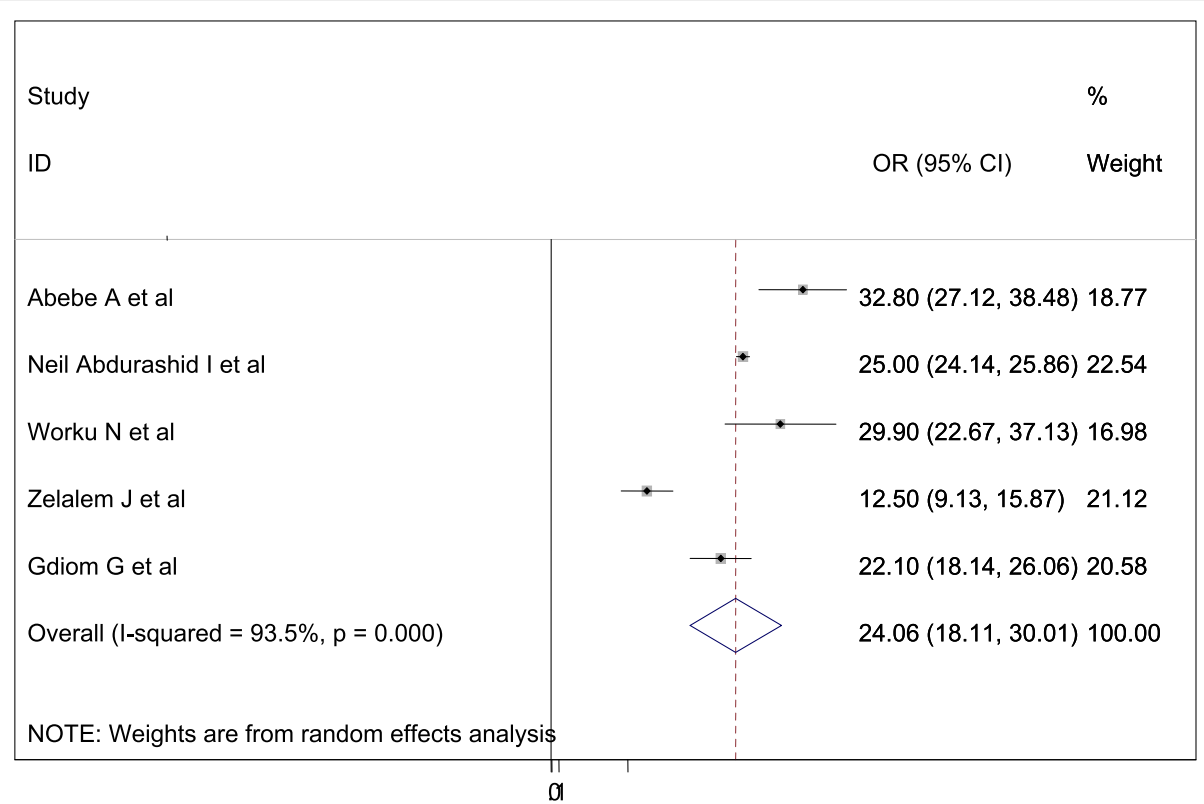

Fig. 2 Forest plot displaying the pooled prevalence of perinatal asphyxia in Ethiopia

conducted in India [30, 31] in Iraq [32], and Uganda [26]. The possible reason could be because the intrapartum inhalation of meconium-stained amniotic fluid resulted in chemical pneumonitis with inflammation of the pulmonary tissues, mechanical obstruction of airways, and pulmonary air leak, inducing hypoxia [33].

Newborns delivered with assisted instrumental deliveries 4.04 times $(\mathrm{OR}=4.04,95 \% \mathrm{CI}=2.48,6.60)$ more likely to develop perinatal asphyxia as compared to with neonates delivered spontaneously. This study finding is supported by the study done in India [30] and Iran [34]. The possible explanation for this might be because instrumental delivery causing birth trauma leads to asphyxia. Instrumental deliveries may affect different locations of cranial hemorrhage; subdural, subarachnoid, intra-parenchyma or intra-ventricular due to exerting pressure of a vacuum and forceps extractors can cause brain bleeds on the cranium contributing to intracranial hemorrhage resulting birth asphyxia, bleeding diathesis, infection, and vascular anomalies. As evidenced by a piece of literature, the incidence of traumatic intracranial hemorrhage of six per 1000 live neonates born than spontaneously had born [35].

The odds of birth asphyxia among babies born to mothers with prolonged labor were about 2.79 times

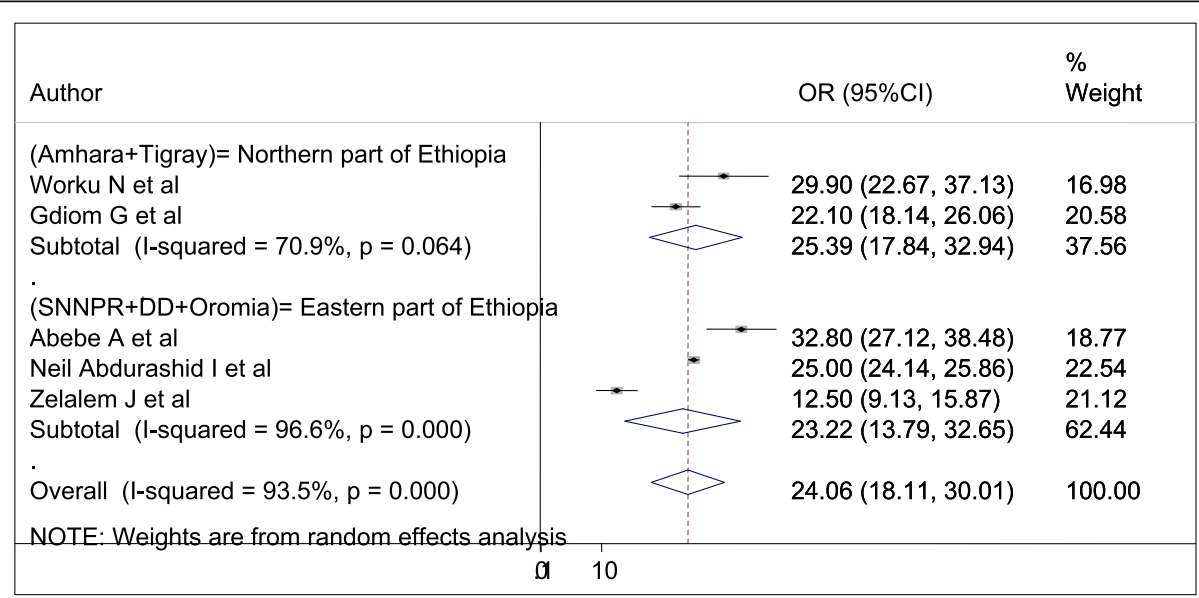

DD: Dire Dawa

Fig. 3 Forest plot of the sub group analysis based on the study area (region) 




Fig. 4 Forest plot of the sub group analysis based on the sample size of the study

$(\mathrm{OR}=2.79, \quad 95 \% \mathrm{CI}=1.98,3.93)$ higher than their counterparts. The finding of this study is consistent with the study done in Bangladesh [36], Cameroon [37], Nigeria [20] and Sweden [38]. This could be because if the labor does not progress normally, the laboring mother may experience serious complications, such as dehydration, exhaustion, or rupture of the uterus [39] and if labor becomes prolonged, there is a high chance of the fetus to become distressed.
Despite this, there is a standard guideline for neonatal resuscitation; the death of neonatal mortality is increasing in Ethiopia. In the Ethiopian context with the adoption of the WHO neonatal resuscitation procedures are employed. Initiate cardiac massage at a rate of 120 beats/min, intubation and continuous positive pressure ventilation with $100 \%$ of oxygen at a rate of 40 breaths per minute, observe for improvements intermittently (initiation of spontaneous respiration; color, heart rate), administer normal saline and sodium bicarbonate to



Fig. 5 Funnel plot test for publication bias for perinatal asphyxia in Ethiopia 


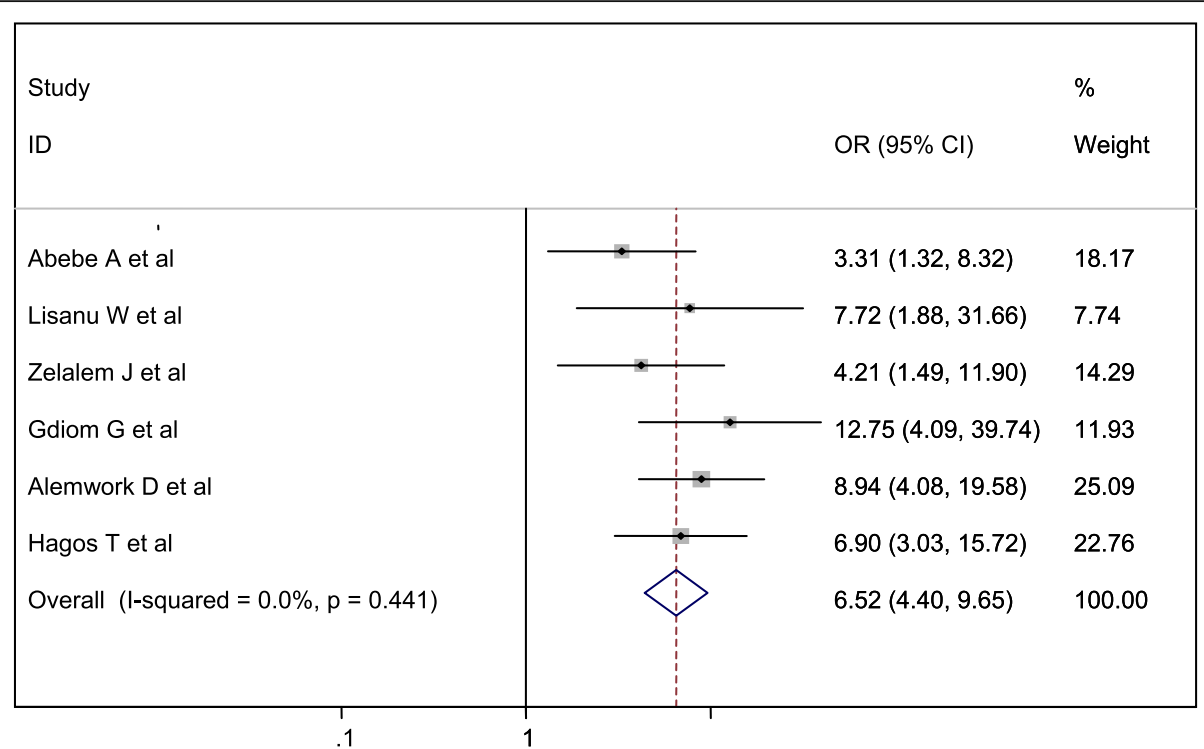

Fig. 6 Forest plot displaying the association between low birth weight and perinatal asphyxia in Ethiopia

counter acidosis and shock respectively), and adrenalin through endotracheal tube were the procedures for neonatal resuscitation [40].

This systematic review and meta-analysis had significant heterogeneity which may prone to publication bias. This might be due to the sample size of each study, the nature of the study design, the study setting or region. Despite this review has marked heterogeneity, subgroup analysis was computed using study setting and sample size and showed improvement of marked heterogeneity to a moderate extent, resulting in reducing the having the chance of publication bias.

\section{Limitation}

This systematic review and meta-analysis revealed to estimate the national burden of perinatal asphyxia. However, we were unable to find any neonatal resuscitation measures and effectiveness from the single studies to show the national burden in this review. Including papers only published by the English

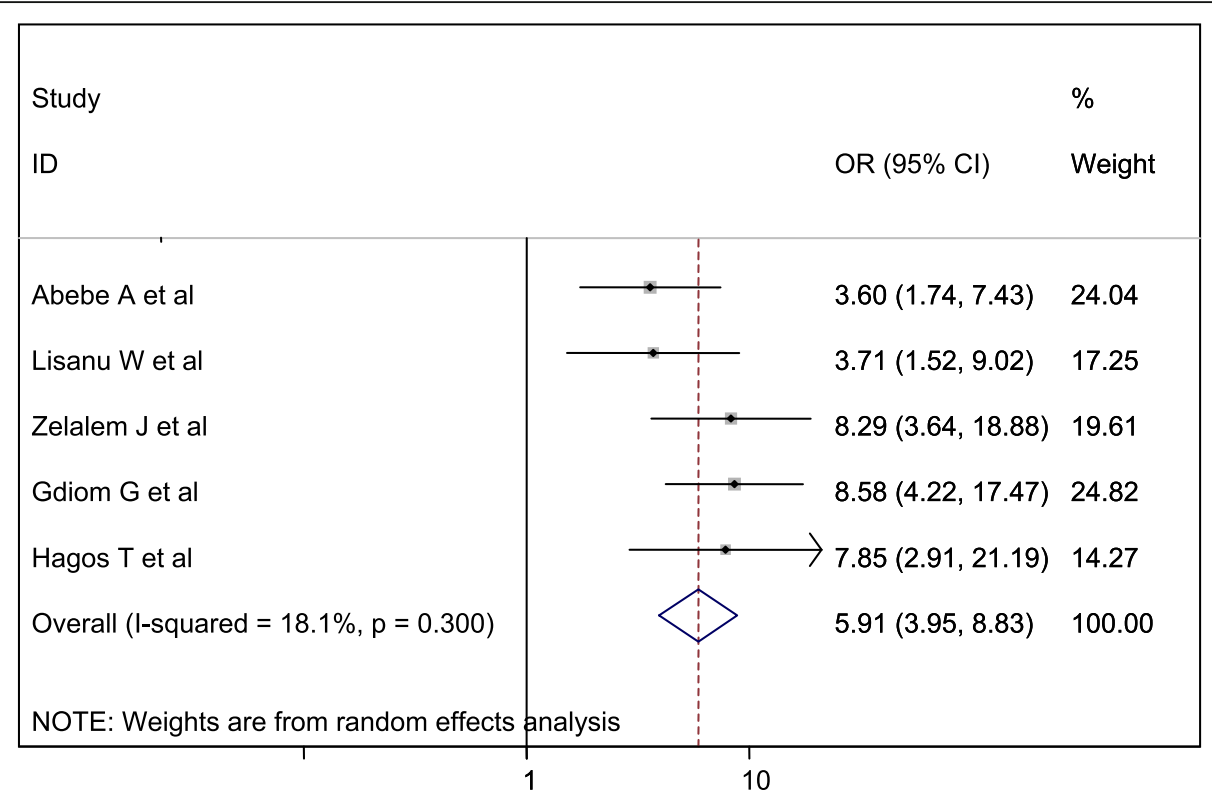

Fig. 7 Forest plot displaying the association between MSAF and perinatal asphyxia in Ethiopia 




Fig. 8 Forest plot displaying the association between assisted instrumental delivery and perinatal asphyxia in Ethiopia

language and accessing only hospital-based studies was the restraint of the study. It might lack national representativeness because no data were from all regions. Besides, this study included smaller studies (those with larger standard errors) tend to have a chance of having publication bias.

\section{Conclusion}

The overall pooled prevalence of perinatal asphyxia was remarkably higher. Prolonged labor, meconium-stained amniotic fluid, instrumental deliveries, and low birth weight were the determinants factors of perinatal asphyxia in Ethiopia. Therefore, efforts might be made to improve the quality of intrapartum care services to prevent labor, delivery and fetal complications and to identify and make a strict follow up of mothers with meconium-stained amniotic fluid. Addressing and identifying determinants and magnitudes of hypoxic-ischemic encephalopathy may improve the implementation of the WHO standard guideline effectively and consistently.

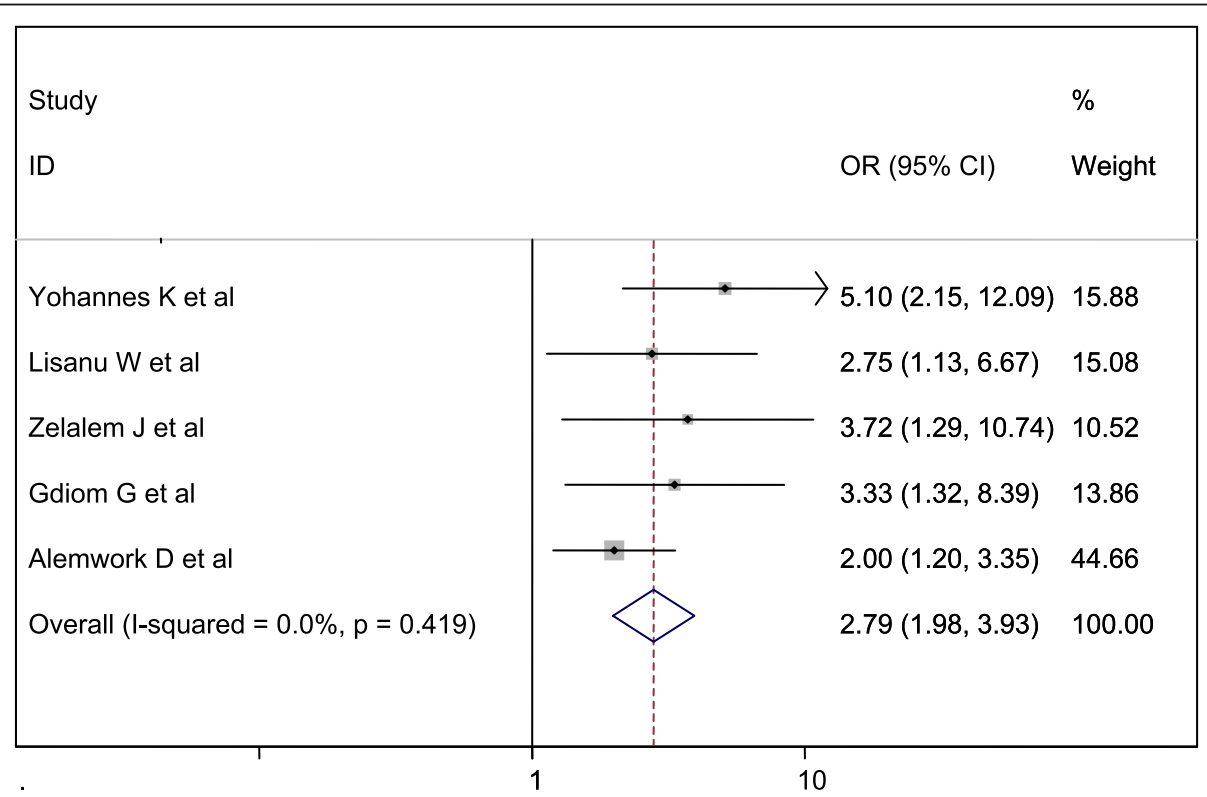

Fig. 9 Forest plot displaying the association between prolonged labor and perinatal asphyxia in Ethiopia 


\section{Abbreviations}

Cl: Confidence Interval; DD: Dire Dawa; MSAF: Meconium stained amniotic fluid; OR: Odds Ratio; PNA: Perinatal asphyxia; WHO: World Health Organization

\section{Acknowledgments}

Not applicable.

\section{Authors' contribution}

GG \& FW involved in the design, selection of articles, data extraction, statistical analysis. GG, FW, and SL were involved in manuscript writing. All authors read and approved the final draft of the manuscript.

\section{Funding}

No funding was obtained for this study.

\section{Availability of data and materials}

All related data has been presented within the manuscript. The dataset supporting the conclusions of this article is available from the authors on request.

\section{Ethics approval and consent to participate}

Not applicable.

\section{Consent for publication}

Not applicable.

\section{Competing interests}

All authors declare that they have no competing interests.

\section{Author details}

'Department of Midwifery, College of Medicine and Health Sciences, Bahir Dar University, Bahir Dar, Ethiopia. ${ }^{2}$ Department of Midwifery, College of Health Sciences, Woldia University, Woldia, Ethiopia.

\section{Received: 28 October 2019 Accepted: 17 March 2020}

\section{Published online: 24 March 2020}

\section{References}

1. WHO. Word Health Statistics: Part II Global health indicators 2015. Available form: http://wwwwhoint/whosis/whostat/EN_WHS2011Fullpdf Accessed 20 Nov 2016.

2. WHO. Word health statistics: part I global health indicators; 2015. Available from: (http://wwwwhoint/whosis/whostat/EN_WHS2011Fullpdf) Accessed 20 Nov 2016.

3. Aslam HMSS, Afzal R, Iqbal U, Saleem SM, Shaikh MW, Shahid N. Risk factors of birth asphyxia in civil hospital Karachi, Dow University of health sciences. Ital J Pediatr. 2014;40(94):1824-7288.

4. WHO. World health statistics;2013. Available from:http://wwwwhoint/ publications/world-health-statistics /2013/en/. 2013.

5. Haider BABZ. Birth asphyxia in developing countries: currentstatus and public health implications. Curr Probl Pediatr Andadolesc Health Care. 2006; 5(36):178-88.

6. Belachew T, Joseph J. Birth asphyxia and associated factors among newborns delivered in Jimma zone public hospitals, Southwest Ethiopia: a cross-sectional study. J Midwifery Reprod Health. 2018;6(2):1289-95.

7. $F M O H ~ U$. Countdown to a healthier Ethiopia: building onsuccesses to accelerate newborn survival; 2015. Available from: (wwwephigovet) Accessed 20 Nov 2016

8. WHO. Global health observatory data repository world health organization; 2015. Available from: http://appswhoint/ghodata/ Accessed 24 Nov 2016.

9. al Le. Distribution of causes of neonatal and under-five deaths, CHERG/ WHO/UNICEF. Lancet. 2014. 2014;388:3027-3035.

10. United Nations Children's Fund WHO, World Bank and United Nations. Levels \& Trends in Child Mortality Report 2015. New York (NY), Geneva and Washington (DC). Available from: http://wwwuniceforg/publications/ flesSept 15pdf Accessed 28 Dec 2016; 2016.

11. Sendeku FW, Azeze GG, Fenta SL. Adherence to iron-folic acid supplementation among pregnant women in Ethiopia: a systematic review and meta-analysis. BMC Pregnancy Childbirth. 2020;20(1):1-9.
12. Long M, Brandon DH. Induced hypothermia for neonates with hypoxicischemic encephalopathy. J Obstet Gynecol Neonatal Nurs. 2007:36:293-8.

13. Wosenu L, Worku AG, Teshome DF, Gelagay AA. Determinants of birth asphyxia among live birth newborns in University of Gondar referral hospital, northwest Ethiopia: A case-control study. PLOS One. 2018;13(9):1-12.

14. Wayessa ZJ, (MSC) TBPaJJM. Birth asphyxia and associated factors among newborns delivered in Jimma zone public hospitals, Southwest Ethiopia: A cross-sectional study. J Midwifery Reprod Health. 2018;6(2):1289-95.

15. Abebe Alemu GM, Aberaand GB, Damte A. Prevalence and associated factors of perinatal asphyxia among newborns in Dilla University referral hospital, southern Ethiopia. Pediatric Health Med Ther. 2017;10:69-74.

16. GdiomGebreheat T, Kiros D, Teame H, Etsay N, Welu G, Abraha D. Prevalence and Associated Factors of Perinatal Asphyxia among Neonates in General Hospitals of Tigray, Ethiopia. BioMed Res Int. 2018:1-7.

17. Meshesha AD, Azage M, Worku E, Bogale GG. Determinants of Birth Asphyxia Among Newborns in Referral Hospitals of Amhara National Regional State, Ethiopia. Pediatric Health, Medicine and Therapeutics. 2020;11:1-12.

18. Tasew H, Zemicheal M, Teklay G, Mariye T, Ayele E. Risk factors of birth asphyxia among newborns in public hospitals of central zone, Tigray, Ethiopia 2018. BMC Res Notes. 2018:11(1):496.

19. Yohannes Kibret1 GHaKA. Determinants of Birth-Asphyxia among Newborns in Dessie Town Hospitals, North-Central Ethiopia. Int I Sex Health Reprod Health Care. 2018;1(1):1-12

20. Bilkisu Garba llah MSA, Musa A. Muyideen bimbo Adelakun, Akeem Oladiran Adeniji, Taofik Kolawole prevalence and risk factors for perinatal asphyxia as seen at a specialist hospital in Gusau, Nigeria Sub-Suharan. Afr J Med. 2015;2(2):64-9.

21. Sepeku AKT. Treatment outcomes of neonatal asphyxia at a national hospital in Dar Es Salaam, Tanzania. Afr J Nurs Midwifery. 2011;13:43-56.

22. Solayman M, Hoque S, Akber T, Islam MI, Islam MA. Prevalence of perinatal asphyxia with evaluation of associated risk factors in a rural tertiary level hospital. KYAMC J. 2017:8(1):43-8.

23. Apirak Nguanboonma SS. Prevalence and Risk Factors of Birth Asphyxia among Elderly Gravidarum. Thai J Obstet Gynaeco. 2012-2016:27(1):29-37.

24. Boskabadi HAF, Doosti H, Zakerihamidi M. Assessment of Risk Factors and 500 Prognosis in Asphyxiated Infants. Iran J Pediatrics. 2015;25(4):1-17.

25. Kuama DM. Prevalence \& Risk factors for Birth Asphyxia in Goroka Provincial Hospital. 2017:1-22.

26. Kaye D. Antenatal and intrapartum risk factors for birth asphyxia among emergency obstetric referrals in Mulago hospital, Kampala, Uganda. East Afr Med J. 2003;80(3):140-3.

27. MT U. Risk factors for birth asphyxia. Folia Medica Indones. 2011:47(4):211-4

28. Yustina Purwaningsih YLRD, Indarto D, Murti B. Factors associated with newborn asphyxia at Dr. Harjono hospital, Ponorogo, East Java. J Matern Child Health. 2018;3(4):287-93.

29. Rachatapantanakorn OTP, Chaisuksant Y. Factors associated with birth asphyxia in Pattani hospital, Thailand. Songklanagarind Med J. 2005;23(1):17-27.

30. Bahubali Gane VBB, Rao R, Nandakumar S, Adhisivam B, Joy R, Prasad P, Shruti S. Antenatal and intrapartum risk factors for perinatal asphyxia in India: a case control study. Curr Peditr Res. 2013;17(2):119-22.

31. Veerendra Mehar NA, Agarwal A, Agarwal S, Dubey N, Kumawat H. Meconium-stained amniotic fluid as a potential risk factor for perinatal asphyxia:India. J Clin Neonatol. 2015:5(3):157-61.

32. Sahib HS. Risk factors of perinatal asphyxia: a study at Al-Diwaniya maternity and children teaching hospital. Muthanna Med J. 2015:2(2):50-7.

33. Yurdakök M. Meconium aspiration syndrome: do we know? Turk J Pediatr. 2011;53(2):121.

34. al KAe. Perinatal Factors Leading to Birth Asphyxia among Term Newborns. J Pediatrics. 2014;24(5):637-42.

35. Ferraz A, Nunes F, Resende C, Almeida MC, Taborda A. Complicaciones neonatales a corto plazo de los partos por ventosa. Estudio caso-control. In: Anales de Pediatría, vol. 91, No. 6: Elsevier Doyma; 2019. p. 378-85.

36. Shireen N, Nahar N, Mollah AH. Risk factors and short-term outcome of birth asphyxiated babies in Dhaka medical college hospital. Bangladesh J Child Health. 2009;33(3):83-9.

37. Chiabi ANS, Evelyne M, Nodem S, Mbuagbaw L, Mbonda E. Risk factors for birth asphyxia in an urban health facility in Cameroon. Iran J Child Neurol. 2013;7(3):46.

38. Altman M, Sandström A, Petersson G, Frisell T, Cnattingius S, Stephansson O Prolonged second stage of labor is associated with low Apgar score. Eur Epidemiol. 2015;30(11):1209-15. 
39. Revicky VMA, Mukhopadhyay S, Mahmood T. A case series of uterine rupture: lessons to be learned for future clinical practice. J Obstet Gynecol. 2012;62(6):665-73.

40. World Health Organization. Guidelines on basic newborn resuscitation. 2012.

\section{Publisher's Note}

Springer Nature remains neutral with regard to jurisdictional claims in published maps and institutional affiliations.

\section{Ready to submit your research? Choose BMC and benefit from:}

- fast, convenient online submission

- thorough peer review by experienced researchers in your field

- rapid publication on acceptance

- support for research data, including large and complex data types

- gold Open Access which fosters wider collaboration and increased citations

- maximum visibility for your research: over $100 \mathrm{M}$ website views per year

At $\mathrm{BMC}$, research is always in progress. 